\title{
Novel Entire Top Surface Planar Leaky Wave Antenna
}

\author{
Jan Zehentner, Jan Machac, Pavel Zabloudil \\ Czech Technical University in Prague, \\ Technicka 2, 16627 Prague, Czech Republic \\ zehentner@fel.cvut.cz \\ machac@fel.cvut.cz \\ zabli@centrum.cz
}

\begin{abstract}
This paper presents the application of a flat waveguide with a longitudinal slot in the middle of its wider wall for the design of a leaky wave antenna. The antenna is fed from a coaxial connector on its bottom, and provides a totally flat top surface and a radiation pattern with better suppression of side lobes than in the case of an antenna fed via a CPW patch. The antenna radiates into one relatively narrow main beam. The antenna characteristics and radiation aspects are discussed here. Its measured characteristics are in accord with those predicted by simulation.
\end{abstract}

\section{INTRODUCTION}

Low profile planar antennas are suitable for a number of applications. This is because they do not perturb the external surface of the bodies on which they are mounted, e.g., cars, airplanes. Planar antennas save space, are cheap, can be easily fabricated and are therefore suitable for mass production. These antennas have therefore been of great interest to researchers and designers for several decades. Many kinds of planar antennas are already known. Their operation is based on two main mechanisms of radiation. The radiation of resonant structures is produced by a standing wave. Such radiators have only a narrow frequency band [1]. However, the radiation due to a wave traveling along a transmission line offers a wider frequency band. Antennas exploiting the traveling wave on slotted waveguides were designed fifty years ago [2] - [4]. Very sophisticated leaky wave antennas based on the stub-loaded rectangular waveguide were reported in [5], [6]. They enable independent adjustment of the beamwidth and the angle of maximum radiation. The drawback is that they are not flat.

All open transmission lines can leak power in some frequency bands and can therefore be used as leaky wave antennas. The first microstrip leaky wave antenna was proposed in [7], and the first slotline leaky wave antenna in [8]. We have successively investigated planar slotted transmission lines in order to find one leaking power only into the half-space. Our studies have included the slotline, the conductor-backed slotline and the inverted conductor-backed slotline [9]. However, all of these exhibit not only the required leakage into the space, but also undesirable leakage into the substrate. Consequently, a transmission line suitable for the design of a planar leaky wave antenna must have a substrate with finite width and thickness, shielded on the side and bottom walls and having a longitudinal slot on the top. A flat slotted waveguide (FSW) [10] meets all these demands. Finally we designed and fabricated an FSW leaky wave antenna fed through a CPW terminated by a narrow patch [11]. This feeder however violated the requirement of antenna surface planarity due to the presence of the coaxial connector axially coupled to the feeding CPW. At the same time, we do not like the idea of an additional absorbing shield positioned around the CPW patch feeding this antenna [11], as it is not planar at all.

Consequently a modified FSW leaky wave antenna is proposed in this paper. This antenna is fed through a coaxial connector and the transition from the coaxial line to a waveguide of a rectangular cross-section [12]. Consequently, the top antenna surface is flat. The measured antenna characteristics, including the radiation pattern, are in good accord with those predicted by the EM solver.

\section{Design OF THE ANTENNA}

The FSW can be viewed as a conductor-backed slotline with a finitely wide substrate metallized on its side walls. The cross-section of the line, with proportions and co-ordinates, is shown in Fig. 1. When $b$ is substantially greater than $h$ we have a flat waveguide with a slot cut in the wider wall of the waveguide parallel to its longitudinal axis. In our experiment we used cheap plexiglass substrate $8 \mathrm{~mm}$ in thickness with permittivity $\varepsilon_{\mathrm{r} 2}=2.6$. To generate effectively a space leaky wave in the frequency band between 4 and $5 \mathrm{GHz}$ we chose according to [11] the line dimensions $\mathrm{b}=38 \mathrm{~mm}, \mathrm{~h}=8 \mathrm{~mm}$, $\mathrm{w}=25 \mathrm{~mm}$.

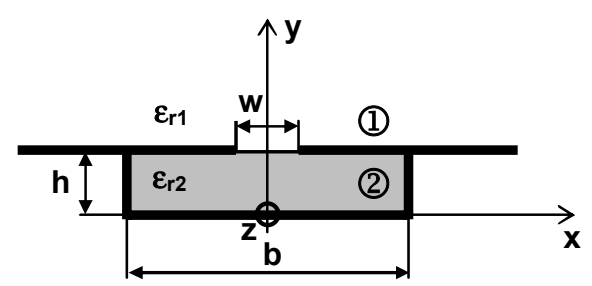

Fig. 1 Cross-section of the flat slotted waveguide.

The antenna presented in [11] is fed axially via a coaxial connector and the CPW terminated by a narrow patch, as shown in the antenna layout in Fig. 2a. The main drawback of this structure is perturbation of the planarity of the top antenna 
surface by the coaxial connector. Moreover this feeder influences the radiation of the antenna.

The new antenna with the top layout shown in Fig. $2 \mathrm{~b}$ is fed from the bottom via a coaxial line and the $\mathrm{T}$ shape adapter to the waveguide of rectangular cross-section [12]. The transition from this waveguide to the FSW is done by the slot taper shown in Fig. 2b. The cross-section of the transition from the coaxial line to the waveguide is drawn in Fig. 2c. This new feeder ensures excitation of the modes with odd symmetry on the FSW, which leak into space. The dimensions of the antenna feeder and the angle of the slot opening were optimized by the CST Microwave Studio (CST MWS), targeting the lowest $\mathrm{S}_{11}$ in the above-mentioned frequency band. The length and width of the antenna, and the FSW termination, determine the shape of the radiation pattern. The waveguide is short-circuited at the side of the feeder and the FSW is open-circuited at its end. The antenna length $l$ of 450 $\mathrm{mm}$ follows from the requirement that the amplitude of the odd space leaky mode at the end of the antenna should be $5 \%$ of its magnitude on the slot input. The final antenna width $g$ was chosen five times wider than the FSW width $b$. The angle of the slot taper $\alpha=28^{\circ}$.
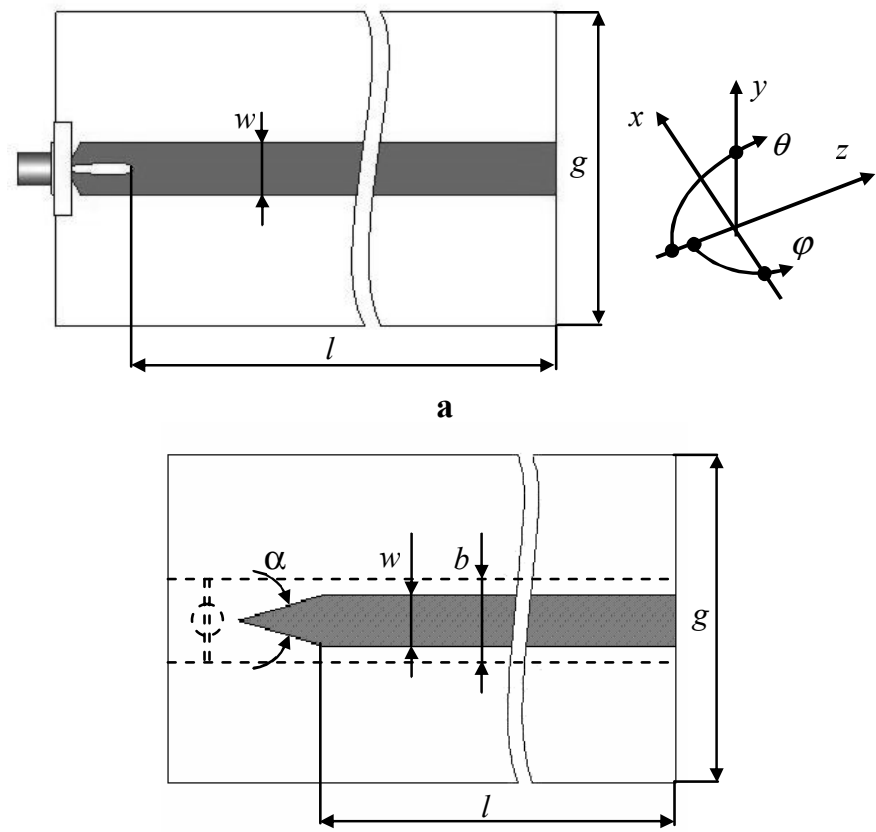

b

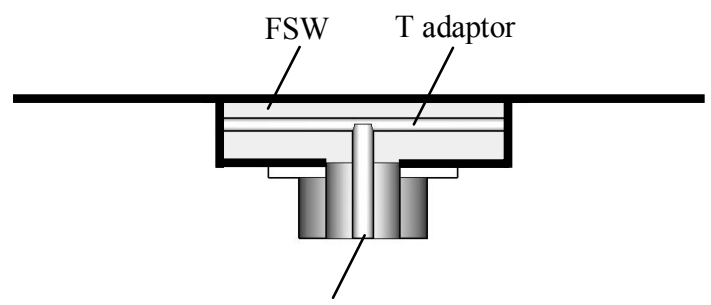

coaxial connector

c

Fig. 2 Layout of the FSW leaky wave antenna, fed via CPW (a), fed from the bottom (b), cross-section of a coupling from a coaxial connector to FSW (c).
Calculating the antenna circuit and radiation parameters, we set the conductivity of aluminum $\sigma=3.5 \cdot 10^{7} \mathrm{~S} / \mathrm{m}$, and the loss factor of the plexiglass $\operatorname{tg} \delta=0.01$ at $4.75 \mathrm{GHz}$.

\section{MEASUREMENTS AND DISCUSSION}

Let us first review briefly the characteristics of the FSW leaky wave antenna fed by the CPW presented in [11], for the purpose of comparison. Due to the very simple antenna fabrication technology the real proportions of the CPW and the feeding patch differ from the theoretically designed values. Therefore all results presented below, both computed and measured, consider the real size of the antenna and its feeder structure. The measured frequency dependent value $\left|\mathrm{S}_{11}\right|$ and the value calculated by CST MWS are plotted in Fig. 3. The frequency band at reflection losses lower than $-10 \mathrm{~dB}$ is $1 \mathrm{GHz}$ from 4.2 to $5.2 \mathrm{GHz}$.

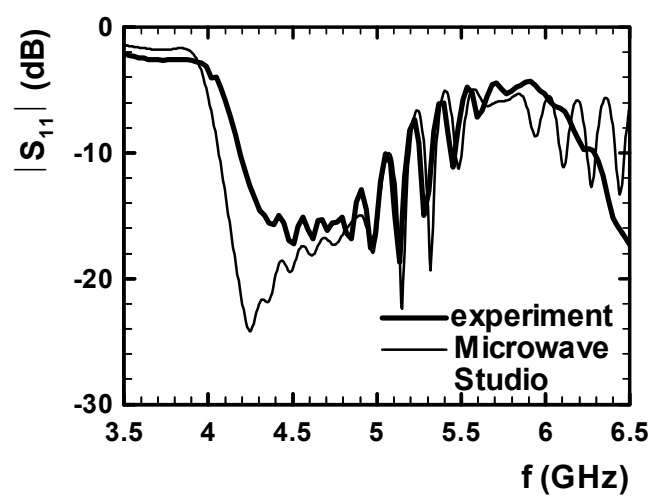

Fig. 3 Measured and calculated return losses of the FSW antenna with the CPW feeding network.

The antenna radiation patterns $\left|E_{\theta}\right|$ measured in the vertical (yz)-plane and calculated by CST MWS at the frequency 4.75 $\mathrm{GHz}$ are plotted in Fig. 4. The radiation patterns are drawn in the spherical coordinates shown in Fig. 2. The main lobe tilts towards the $\mathrm{z}$ axis from $19^{\circ}$, read from the horizontal (xz)plane at $4.2 \mathrm{GHz}$, to $9^{\circ}$ at $5.2 \mathrm{GHz}$. The measured antenna gain at $4.75 \mathrm{GHz}$ is $14.2 \mathrm{~dB}$ and the calculated directivity is $15.47 \mathrm{~dB}$.

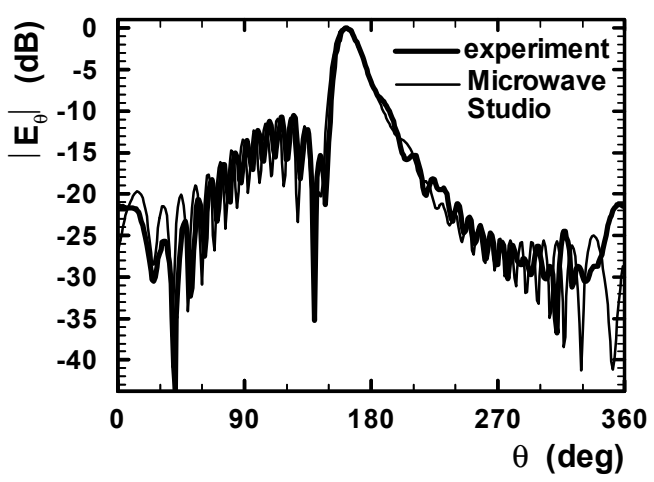

Fig. 4 Radiation patterns of the FSW antenna from Fig. 2 a at $4.75 \mathrm{GHz}$ in the (yz)-plane. 
The FSW antenna with the new feeding circuit shown in Figs. 2b, 2c was investigated both numerically and experimentally. The frequency dependent reflection coefficient $\left|S_{11}\right|$ is plotted in Fig. 5, while $S_{11}$ is shown in the Smith Chart in Fig. 6. The frequency band corresponding to reflection coefficient lower than $-10 \mathrm{~dB}$ is $1 \mathrm{GHz}$ from 4 to 5 $\mathrm{GHz}$. This is very similar to the frequency band of the original FSW leaky wave antenna.

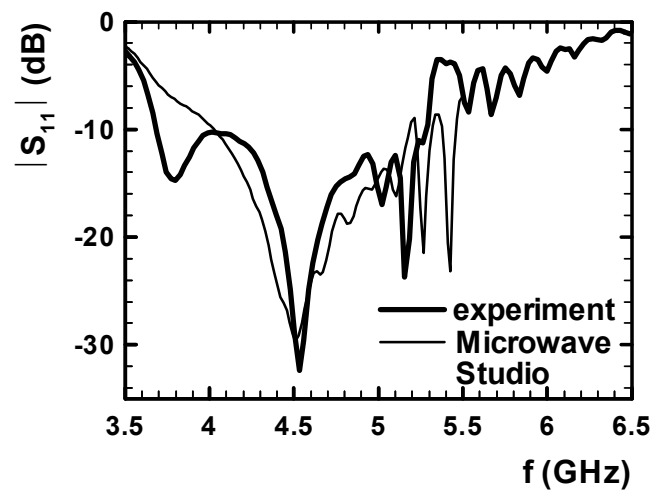

Fig. 5 Measured and calculated return losses of the FSW antenna fed from the bottom, Fig. 2 b.

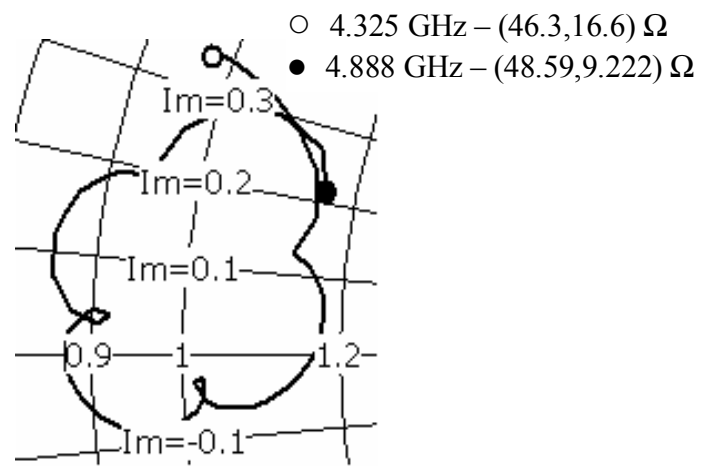

Fig. 6 Calculated reflection coefficient of the FSW antenna fed from the bottom, Fig. 2 b.

The calculated and measured radiation patterns of the antenna fed from the bottom are shown in Fig. 7 at the frequency $4.55 \mathrm{GHz}$ corresponding to the best antenna match. The patterns are taken in the (yz) plane as a function of angle $\theta$. The patterns are normalized to $0 \mathrm{~dB}$ at their maxima. In comparison with the patterns of the original antenna shown in Fig. 3, the antenna presented here has a better side lobe level (SLL), about $-20 \mathrm{~dB}$ below the maximum of radiation. The SLL gets worse with frequency decline from $4.55 \mathrm{GHz}$ both up and down. The declination of the main beam from the antenna plane (xz) is steered by the frequency from $42^{\circ}$ measured at $4 \mathrm{GHz}$ to $14^{\circ}$ at $5 \mathrm{GHz}$. The width of this beam in the vertical (yz) plane, defined in the field decrease by $3 \mathrm{~dB}$ from the maximum value, changes with frequency from $36^{\circ}$ at $4 \mathrm{GHz}$ to $18.5^{\circ}$ at $4.75 \mathrm{GHz}$ and again raises to $22^{\circ}$ at $5 \mathrm{GHz}$. Fig. 7 shows also the cross-polarization, which is about -15 $\mathrm{dB}$ below the maximum of the $E_{\theta}$ field component. The measured antenna gain is $12 \mathrm{~dB}$, i.e., slightly lower than the 14.2 $\mathrm{dB}$ value of the original FSW leaky wave antenna.

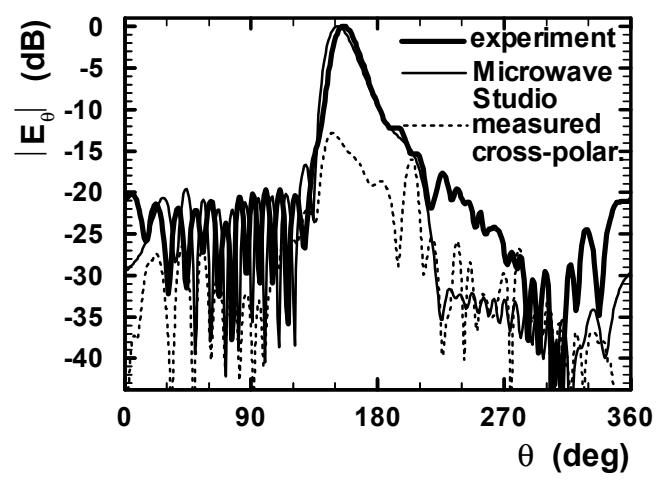

Fig. 7 Calculated and measured radiation patterns of the FSW antenna fed from the bottom, Fig. $2 \mathrm{~b}$ at $4.55 \mathrm{GHz}$ in the (yz)-plane.

Fig. 8 shows the measured and calculated radiation patterns of the present FSW leaky wave antenna plotted as a function of the azimuthal angle $\varphi$ at the frequency $4.55 \mathrm{GHz}$. The pattern is measured at the plane declined from the antenna plane (xz) by an angle of $24^{\circ}$, e.g., in the direction of the main radiation pattern beam shown in Fig. 7 . The radiation pattern calculated by CST MWS is taken on a conical surface comprehending a straight line deflected by the above defined angle from the antenna plane. However, the patterns copy each other well around the main lobe. The radiation pattern is considerably wider in this plane than in the vertical (yz) plane, documented by the calculated $3 \mathrm{D}$ radiation pattern shown in Fig. 9. The $-3 \mathrm{~dB}$ width of the radiation pattern in the azimuth varies from $52^{\circ}$ at $4.55 \mathrm{GHz}$, measured in the direction $24^{\circ}$ above the (xz) antenna plane, to $27^{\circ}$ at $5 \mathrm{GHz}$ measured in the direction $14^{\circ}$ above the antenna plane. Below $4.55 \mathrm{GHz}$ it made no sense to speak about the width of the radiation pattern in the azimuth, as it was split into two lobes. This is documented by the radiation patterns measured in the antenna (xz) plane plotted at $4,4.55$, and $5 \mathrm{GHz}$ in Fig. 10. The continual splitting off of the radiation pattern is shown when the frequency decreases.

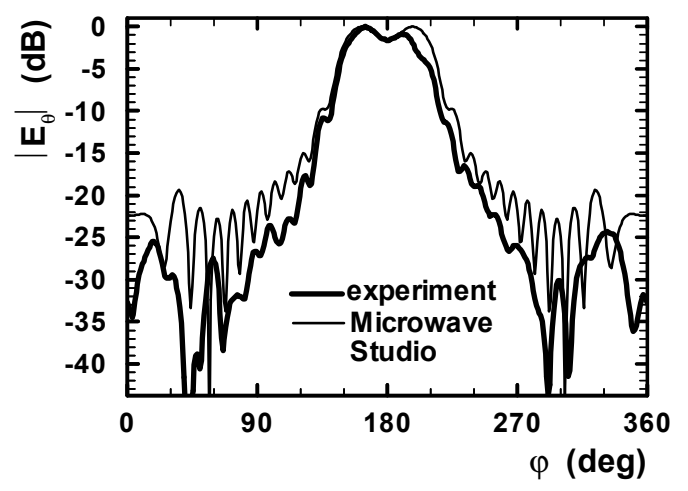

Fig. 8 Calculated and measured radiation patterns of the FSW antenna fed from the bottom, Fig. $2 \mathrm{~b}$ at $4.55 \mathrm{GHz}$ in the plane declined by an angle of $24^{\circ}$ from the azimuthal plane. 


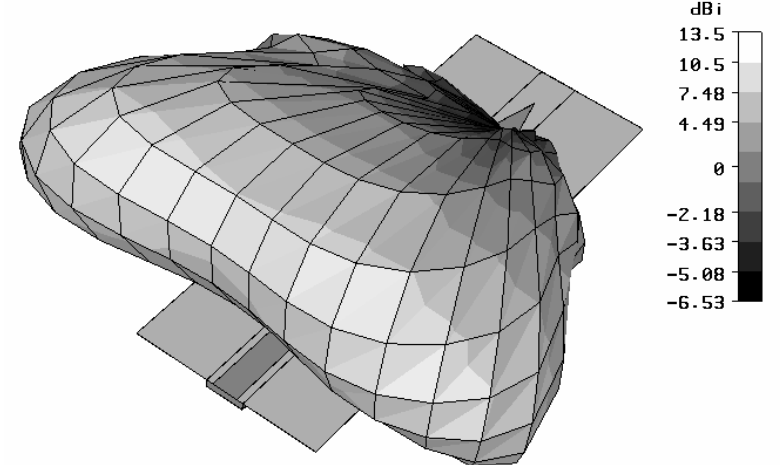

Fig. 9 3D radiation pattern of the FSW antenna at $4.55 \mathrm{GHz}$, calculated by CST MWS

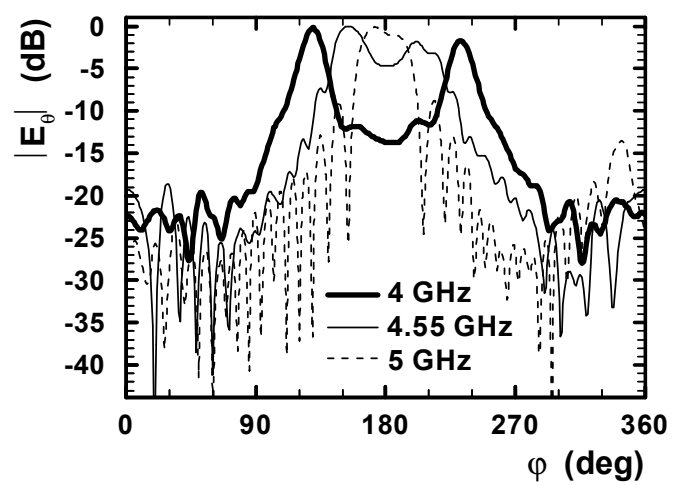

Fig.10 Measured radiation patterns of the FSW antenna fed from the bottom, Fig. 2b, in the antenna plane (xz).

\section{CONCLUSIONS}

A flat slotted waveguide leaky wave antenna was designed and fabricated. CST MWS optimized the antenna layout and the feeding circuit. The great advantage of the new feeder located at the bottom of the antenna is that it ensures that the top surface of the antenna is entirely planar. The feeder circuit consists of the T-shaped adapter from the coaxial line to the flat waveguide of the rectangular cross-section cascaded by the tapered slot transferring the wave in the flat slotted waveguide. The feeder transforms the wave from the coaxial line to the FSW with the same efficiency as does the original feeder consisting of the CPW and its patch. The operation frequency band defined by a reflection coefficient lower than $-10 \mathrm{~dB}$ is from 4 to $5 \mathrm{GHz}$. The antenna radiates one main beam steered in the vertical (yz) plane from $42^{\circ}$ to $14^{\circ}$ when the frequency changes from $4 \mathrm{GHz}$ to $5 \mathrm{GHz}$. The full width at half power of the main antenna beam in the (yz)-plane varies round $20^{\circ}$ from 4.5 to $5 \mathrm{GHz}$. The radiation pattern in the (xz)-plane is remarkably wider than that in the (yz)-plane. The finite proportions of the ground conductors are responsible for radiation below the antenna plane. This portion of the radiation fades away with widening of the ground, identical with the conductive surface of the body on which the antenna is fixed. In comparison with an antenna fed via the $\mathrm{CPW}$, the antenna presented here has a similar frequency band and its radiation pattern is less affected by the odd bound mode simultaneously excited with the leaky mode.
The side lobes are about $20 \mathrm{~dB}$ below the level of the maximum radiation at $4.55 \mathrm{GHz}$. They increase when the frequency is declined from this frequency. Moreover, the main lobe of the radiation pattern is steered by the changing frequency in a wider span of angles than in the case of the CPW fed antenna.

The antenna was investigated at relatively low frequencies, allowing easy access for measuring equipment, and it does not require very high-precision fabrication. The antenna can easily be scaled and redesigned to higher frequencies with reduced dimensions. The large number of parameters facilitates modifications of the antenna design, and it is possible to comply with specific requirements, such as radiation pattern, frequency steering, beam elevation, operation frequency band and gain. More precise fabrication and gold metallization would surely lower the own antenna losses and enhance its gain and efficiency. The FSW leaky wave antenna presented here is entirely planar and is applicable when small angles of beam elevation above a large conducting surface are required.

\section{ACKNOWLEDGEMENT}

This work has been supported by the Grant Agency of the Czech Republic under project 102/06/1106 "Metamaterials, nanostructures and their applications".

\section{REFERENCES}

[1] I. J. Bahl and P. Bhartia, Microstrip Antennas, Dedham: Artech House, 1980.

[2] J. N. Hines, V. H. Rumsey and C. H. Walter, "Traveling-Wave Slot Antennas," Proc. IRE, vol. 41, no. 11, pp. 1624-1631, November 1953.

[3] L. O. Goldstone and A. A. Oliner, "Leaky-Wave Antennas I: Rectangular Waveguides," IRE Trans. Antennas \& Propagation, vol. 7, no. 10, pp. 307-319, October 1959.

[4] F. J. Zurker: "Surface and leaky-wave antennas," in Antenna Engineering Handbook, H. Jasik, ed., New York: McGrew Hill, 1961.

[5] P. Lampariello, F. Frezza, H. Shigesava, M. Tsuji and A. A. Oliner, "A Versatile Leaky-Wave Antenna Based on Stub-loaded Rectangular Waveguide: Part I - Theory," IEEE Transactions on Antennas and Propagation, vol. 46, no. 7, pp. 1032-1041, July 1998.

[6] F. Frezza, P. Lampariello, H. Shigesava, M. Tsuji and A. A. Oliner, "A Versatile Leaky-Wave Antenna Based on Stub-loaded Rectangular Waveguide: Part II - Effects of Flanges and Finite Stub Length," IEEE Transactions on Antennas and Propagation, vol. 46, no. 7, pp. 10421046, July 1998.

[7] W. Menzel: "A New Traveling-Wave Antenna in Microstrip," Arch. Electr. Uebertrag. Tech., Vol. 33, pp. 137-140, April 1979.

[8] J.-W. Sheen and Y.-D. Lin, " Propagation Characteristics of the Slotline First Higher Order Mode," IEEE Transactions on Microwave Theory and Techn., vol. 46, no. 11, pp. 1774-1781, November 1998.

[9] J. Macháč, J. Zehentner and J. Hruška, "Conductor-Backed Slotline Antenna," Proceedings of the 34th European Microwave Conference, vol. 2, pp. 1205-1208, October 2004.

[10] J. Zehentner, J. Macháč and J. Mrkvica, "Flat Waveguide with a Longitudinal Slot," 2005 IEEE MTT-S International Microwave Symposium Digest, TH4C-4, June 2005.

11] J. Zehentner, J. Machac, P. Zabloudil, "Low profile slotted flat waveguide leaky wave antenna“, 2006 IEEE MTT-S International Microwave Symposium Digest, San Francisco, CA, June 2006, TH1A, pp. 1303-1306.

[12] G. L. Ragan, ed., Microwave Transmission Circuits, McGraw-Hill, 1948. 